P34 EVALUATING FAMILIARITY, BARRIERS AND ENABLERS TO HEEADSSS PSYCHOSOCIAL ASSESSMENT IN ADOLESCENTS IN A TERTIARY PAEDIATRIC CENTRE

H Samarendra*, K Sullivan, K Malbon. Paediatrics, Imperial College NHS Foundation Trust, London, UK

\subsection{6/bmjpo-2019-RCPCH-SAHM.38}

The HEEADSSS assessment is a validated tool for assessing wellbeing and identifying psychosocial risk during adolescence. Early identification of concerns enables health professionals to deliver education and implement timely and appropriate interventions. However, there is little research examining clinician familiarity and confidence with taking a psychosocial history using the HEEADSSS assessment tool.

The aims of this study are two-fold (1) Investigate staff familiarity and (2) Evaluate enablers and barriers to psychosocial history taking with the HEEADSSS assessment.

Methods This study employs purposive sampling of multi-disciplinary staff routinely involved in assessment of young people. Participants were asked whether they were familiar with the HEEADSSS tool, and those responding 'Yes' were invited to further discuss perceptions, enablers and barriers through a semi-structured interview. A mix of likert-styled numerical scales, and open-ended questions were asked. Answers were transcribed iteratively for analysis. Basic quantitative methods were applied for likert-based responses. Principles of thematic analysis were used to derive common themes from answers to open ended questions.

Results 20 of 40 participants were unfamiliar with the HEADSSS assessment tool, and only 9/20 interviewees identified HEADSSS as a psychosocial interview. Attitudes regarding the importance of psychosocial assessment varies considerably (3.65/5), as does confidence in using HEEADSSS (3.55/5). Service pressures and concerns about appropriateness were the most commonly cited barriers (table 1). Staff awareness and teaching were identified as enablers to encourage use of HEEADSSS.

\begin{tabular}{lr} 
Abstract P34 Table 1 Factors identified as barriers to use of \\
HEADSSS as a psychosocial assessment & $\mathrm{N}=$ \\
\hline & 9 \\
\hline Service pressures & 9 \\
Environmental constraints & 13 \\
Appropriateness of the & 9 \\
interview & 9 \\
Interview methods itself
\end{tabular}

Conclusion Improving the mental health and wellbeing of young people is identified as a priority for patients and policy-makers. Wider delivery and confidence in taking a psychosocial history using the HEEADSSS assessment tool in secondary care is vital in detecting and addressing needs. This study highlights priorities to promote appropriate practice of psychosocial assessment: (1) training in adolescent health and history-taking; (2) defined management and referral pathways. Trust-wide stakeholder engagement with easily accessible interactive teaching and simulation training are proposed as initial enabling methods.

\section{P35 PEDIATRIC PRACTICE PATTERNS OF THE ASSESSMENT AND TREATMENT OF CHILDREN AND ADOLESCENTS WITH MEDICALLY UNEXPLAINED SYMPTOMS - A DANISH NATIONAL SURVEY}

${ }^{1} \mathrm{~K}$ Kooij ${ }^{*},{ }^{1} \mathrm{GK}$ Teilmann, ${ }^{2} \mathrm{CU}$ Rask. ${ }^{1}$ Børne- og Ungeafdelingen, Nordsjaellands Hospital, Hillerød,Denmark; 'Børne- og Ungdomspsykiatrisk Afdeling, Aarhus Universitetshospital, Aarhus, Denmark

\subsection{6/bmjpo-2019-RCPCH-SAHM.39}

Background Children and adolescents with medically unexplained symptoms (MUS) are commonly encountered in the pediatric setting. Still, clinical guidelines and systematic documentation of the content and extent of assessment and treatment of this patient group are lacking.

Aim To explore practice patterns of the assessment and treatment of children and adolescents with MUS in the Danish pediatric setting.

Method A de novo questionnaire with 60 questions was developed. This included questions about current treatment program, available resources, expectations for future patient volume and the experienced need for clinical guidelines. A qualified informant from each of the 19 pediatric departments was identified by the head of the department and invited to complete the questionnaire. Departments were categorized according to their quality of care, based on a multidisciplinary treatment program recommended in internal literature, by assessing the content of current treatment program as well as patterns of collaboration with CAMHS and other health sectors. The results were analyzed using descriptive statistics.

Results All 19 pediatric departments participated. Among these, two had no available treatment program, 7 reported a less comprehensive, but still multidisciplinary program, and 10 reported a more comprehensive, multidisciplinary program.

Eighteen respondents out of 19 (94\%) expect a future increase in pediatric patients with MUS.

Ten of $17(59 \%)$ departments with established treatment reported a recent decrease in resources. Two thirds (67\%) of the respondents express a need for clinical guidelines.

Conclusion To the best of our knowledge, this is the first systematic study on the content and extent of care for patients with MUS in the pediatric setting. Substantial differences with regard to the quality of care to children and adolescents with MUS were observed. The trend of decreasing resources while expecting a future increase in the prevalence of children and adolescents with MUS within the health care system is worrisome. Developing clinical guidelines for assessment and treatment of children and adolescents with MUS, can potentially help to allocate resources and secure evidence-based care in the pediatric setting in general to this patient group. 